\title{
Cardiogenic Shock with Acute Pulmonary Oedema due to Non-ST Elevation Myocardial Infarction (NSTEMI) and Underlying Severe Aortic Valve Stenosis (AS)
}

\author{
Ief Hendrickx ${ }^{1 *}$, Jo Dens ${ }^{1}$ \\ ${ }^{\text {I} F r o ~ Z i e k e n h u i s ~ O o s t-L i m b u r g, ~ S c h i e p s e ~ b o s ~ 6, ~} 3600$ Genk, Belgium
}

Abstract
Presenting a case of a patient with cardiogenic shock with acute pulmonary oedema due to Non-ST Elevation
Myocardial Infarction (NSTEMI) and underlying severe Aortic Valve stenosis (AS). The patient was initially
treated with Bilevel Positive Airway Pressure (BIPAP) and diuretics, followed by intubation because of
respiratory deterioration and Continuous Venovenous Hemofiltration (CVVH) because of metabolic disorders
and ongoing pulmonary oedema. Eventually, the patient's overall condition declined with transfer to the cathlab.
First, an urgent Percutaneous Transluminal Aortic Valvuloplasty (PTAV) was performed, followed by coronary
angiography and Percutaneous Coronary Intervention (PCI) of a dominant Circumflex. Finally, as a ventricular
support device, an Impella was implanted to unload the left ventricle. Despite all the effort, multi organ failure
(MOF) occurred and the patient passed away17 days after admission to the hospital.
Keywords: NSTEMI, Cardiac Care Unit, Coronary Artery.

\section{Case Report}

A 76-year old female patient presented at the Emergency Room (ER) because of acute respiratory distress. She was known with an Ischemic Cerebrovascular Accident (CVA 2018) and a severe aortic valve stenosis (AS - AVA 0.6 $\mathrm{cm}^{2}$ ). Her cardiovascular risk factors included past smoker, insulin-dependent diabetes and hypercholesterolemia. On her initial presentation at the ER, she showed signs of shock with hypotension (BP 85/55 $\mathrm{mmHg}$ ), tachycardia and even desaturation (84\%).

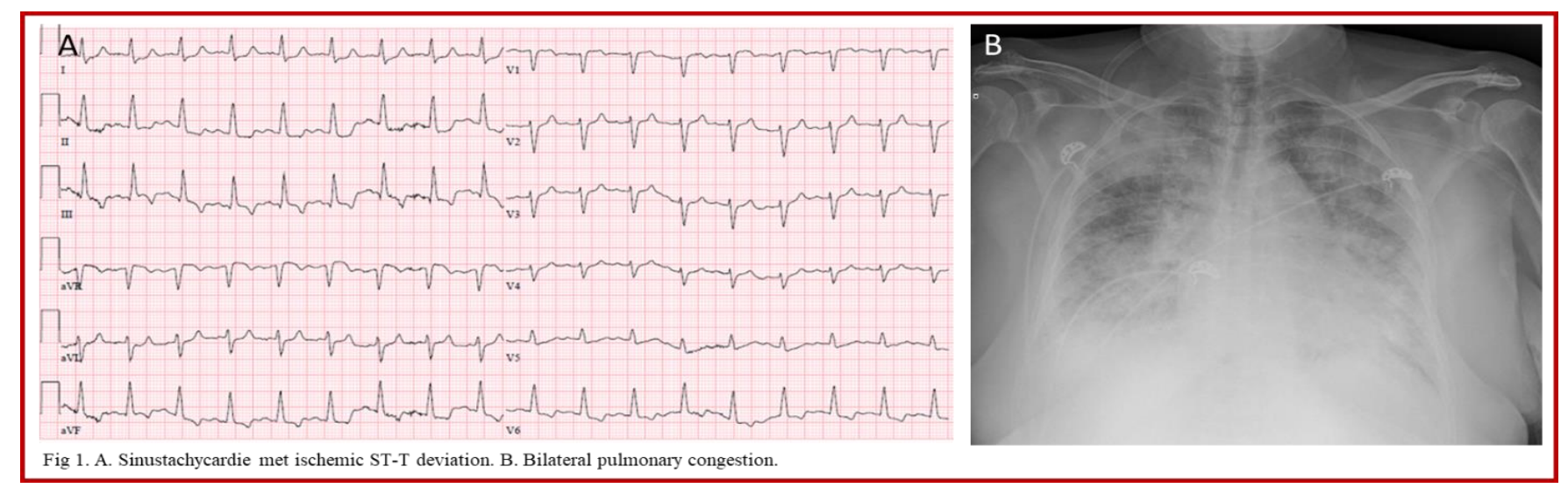

The electrocardiogram (ECG) rendered sinus- tachycardia $110 \mathrm{bpm}$ with ST-T depression in the inferolateral leads with mildly elevated Hs troponin T (168 ng/L - cut-off < $100 \mathrm{ng} / \mathrm{L}$ ) and there was bilateral pulmonary congestion on her chest X-ray (Fig 1A + B).
Corresponding Author: Ief Hendrickx, Ziekenhuis Oost-Limburg, Schiepse bos 6, 3600 Genk, Belgium Received date: November 03, 2019; Accepted date: November 14, 2019; Published date: November 15, 2019.

DOI: https://doi.org/10.31546/JCCCVT.1006 
Bedside transthoracic echocardiography (TTE) showed severely depressed left ventricle function (visual estimation about $15 \%$ ) as a result of diffuse hypokinesia and apical and lateral akinesia and the known underlying severe AS.

Diagnosis of cardiogenic shock with acute pulmonary oedema due to Non-ST Elevation Myocardial Infarction (NSTEMI) and underlying severe AS was made and the patient was admitted to the Cardiac Care Unit (CCU). Therapy with Bilevel Positive Airway Pressure (BIPAP) and diuretics was initiated. Because of respiratory deterioration, the patient was intubated and ventilated. The medical status of the patient further declined with renal failure, anuria, metabolic disorders with hyperkalemia and ongoing pulmonary oedema. Therefore, Continuous Venovenous Hemofiltration $(\mathrm{CVVH})$ was started. Despite all this and support with vasopressors and inotropics, further decline of her cardiac function and overall condition occurred.

The decision for transfer to the cathlab was made. In the assumption that her severe AS was the main trigger of her medical decline, an urgent Percutaneous Transluminal Aortic Valvuloplasty (PTAV) was performed with one successful inflation of a $20 \mathrm{~mm}$ Tyshak balloon (Numed, US) (Fig 2) without right ventricle pacing. Reduction of the transvalvular gradient was obtained from $30 \mathrm{mmHg}$ (during shock) to $5 \mathrm{mmHg}$.

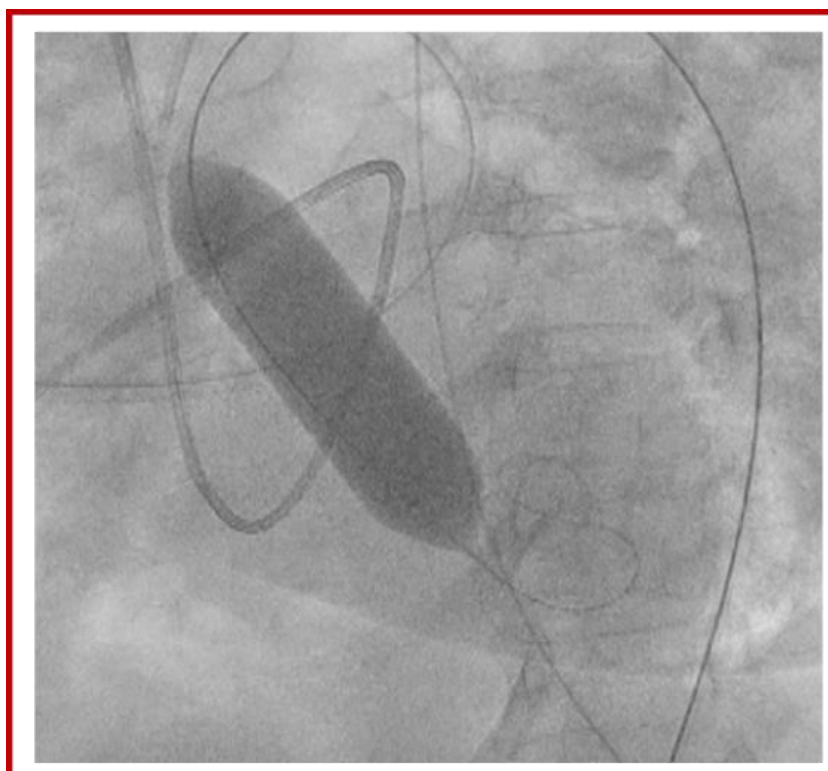

Fig 2. Percutaneous Transluminal Aortic Valvuloplasty

Secondly, coronary angiography was performed. The right coronary artery (RCA) and left anterior descendens (LAD) were free from significant lesions (Fig 3), but the dominant circumflex (Cx) showed a severe stenosis of the proximal segment which was treated with one drug eluting stent $(3.0 \times 16 \mathrm{~mm}$ Cre 8 Evo 3, Alvimedica, Italy) (Fig 4).
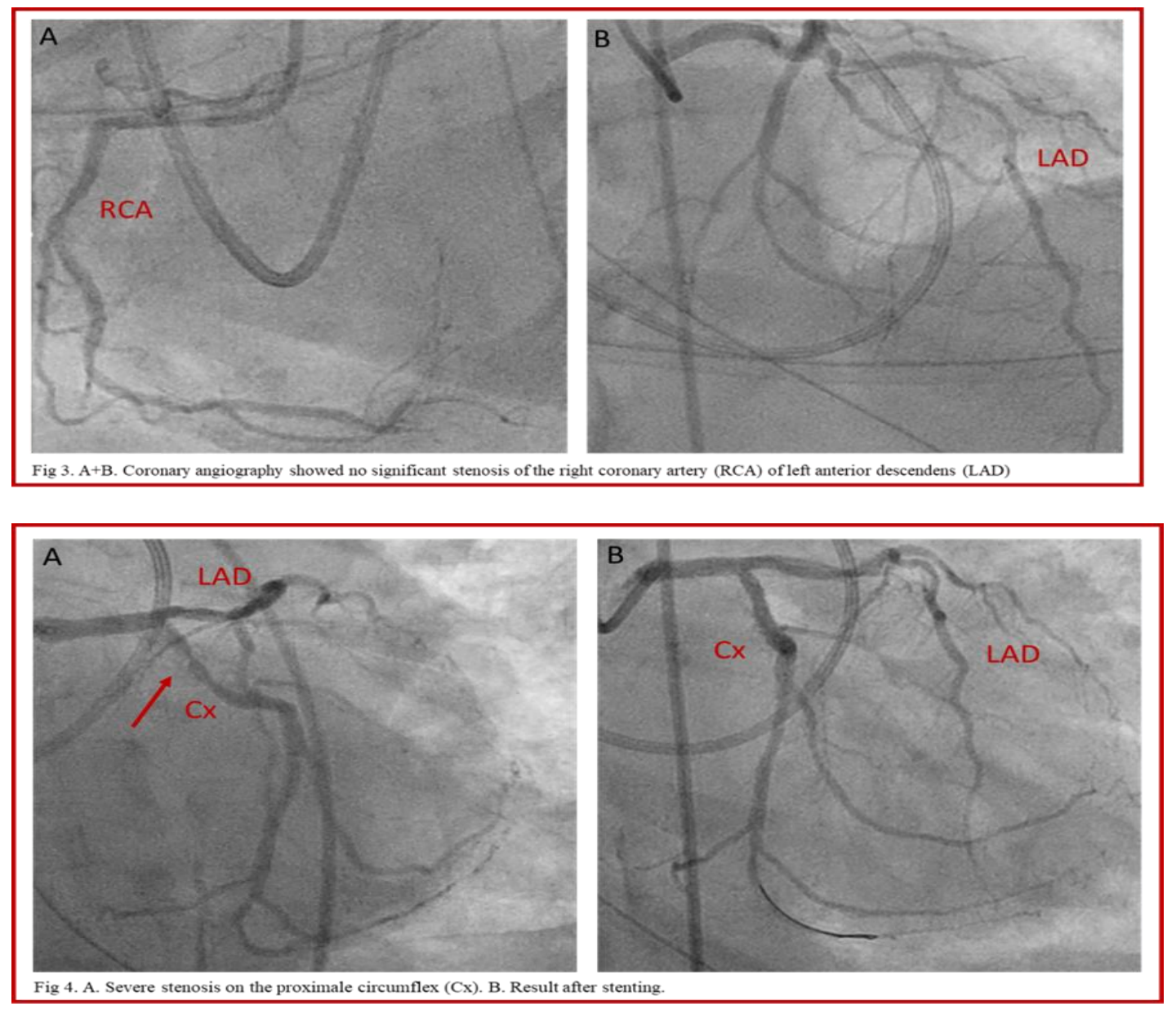
Finally, because of her cardiogenic shock, a ventricular support device Impella CP4 (Abiomed, US) was implanted (Fig 5) with generated flow up to 3,2 liters/minute and the patient was transferred to the $\mathrm{CCU}$ for further care. Two days later, control TTE showed increase in LV function (from 15 to $35 \%$ ). The Impella was removed 3 days after the implantation because of device trombosis, nevertheless the patient remained hemodynamic stable. Despite all the effort, multi organ failure (MOF) occurred with shock liver, rhabdomyolysis, upsloping oxygen demand, etc. The patient passed away 17 days after admission to the hospital.

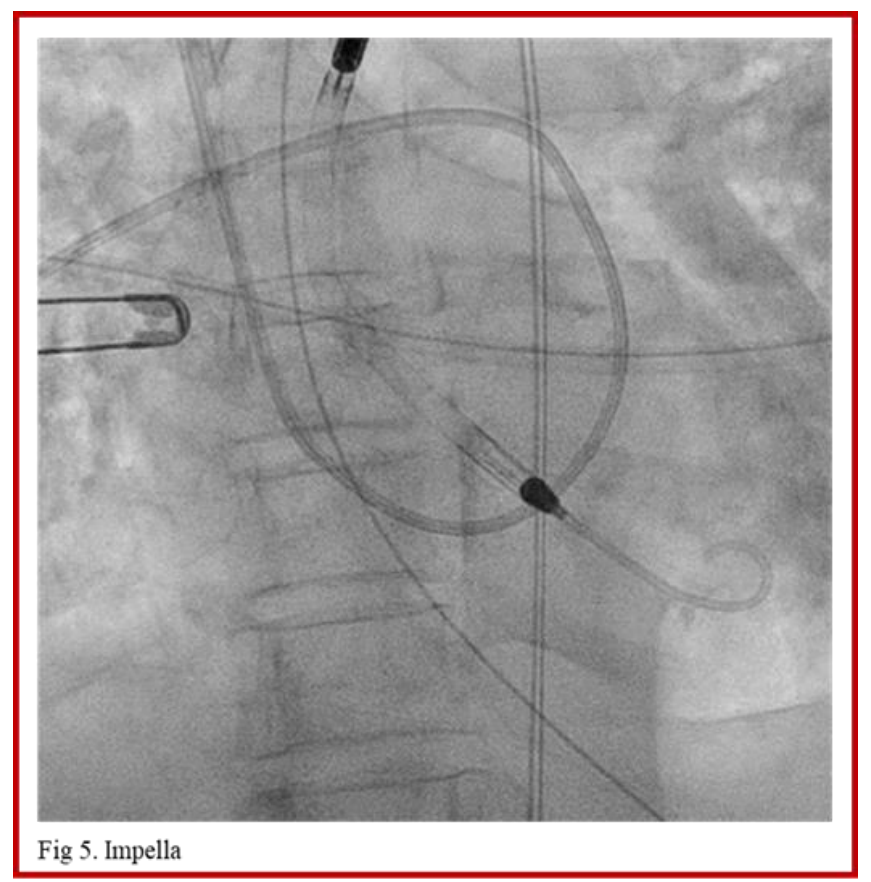

Conflict of Interest: The authors have no conflicts of interest to declare.

Consent for Publication: Written informed consent was obtained from the patient for the publication of this case report. 